OPEN ACCESS

Edited by:

Samar S. Ayache,

Hôpitaux Universitaires Henri

Mondor, France

Reviewed by:

Monica Falautano,

San Raffaele Hospital (IRCCS), Italy

Riley Bove

University of California, San Francisco,

United States

*Correspondence:

Johannes Gehrig

jgehrig@med.uni-frankfurt.de

Specialty section:

This article was submitted to

Multiple Sclerosis and

Neuroimmunology,

a section of the journal

Frontiers in Neurology

Received: 17 December 2021

Accepted: 21 January 2022

Published: 14 February 2022

Citation:

Gehrig J, Bergmann HJ, Fadai L,

Soydaș D, Buschenlange $C$

Naumer MJ, Kaiser J, Frisch S,

Behrens M, Foerch $C$ and Yalachkov $Y$ (2022) Visual Search in Naturalistic Scenes Reveals Impaired Cognitive

Processing Speed in Multiple Sclerosis. Front. Neurol. 13:838178.

doi: 10.3389/fneur.2022.838178

\section{Visual Search in Naturalistic Scenes Reveals Impaired Cognitive Processing Speed in Multiple Sclerosis}

\author{
Johannes Gehrig ${ }^{1 *}$, Heinrich Johannes Bergmann ${ }^{1}$, Laura Fadai ${ }^{1}$, Dilara Soydaş ${ }^{1}$, \\ Christian Buschenlange ${ }^{1}$, Marcus J. Naumer ${ }^{2}$, Jochen Kaiser ${ }^{2}$, Stefan Frisch ${ }^{3,4}$, \\ Marion Behrens ${ }^{1}$, Christian Foerch ${ }^{1}$ and Yavor Yalachkov ${ }^{1}$ \\ ${ }^{1}$ Department of Neurology, University Hospital Frankfurt, Frankfurt, Germany, ${ }^{2}$ Institute of Medical Psychology, \\ Goethe-University, Frankfurt, Germany, ${ }^{3}$ Institute of Psychology, Goethe-University, Frankfurt, Germany, ${ }^{4}$ Department of \\ Gerontopsychiatry, Psychosomatic Medicine, and Psychotherapy, Pfalzklinikum, Klingenmünster, Germany
}

Background: Standardized neuropsychological testing serves to quantify cognitive impairment in multiple sclerosis (MS) patients. However, the exact mechanism underlying the translation of cognitive dysfunction into difficulties in everyday tasks has remained unclear. To answer this question, we tested if MS patients with intact vs. impaired information processing speed measured by the Symbol Digit Modalities Test (SDMT) differ in their visual search behavior during ecologically valid tasks reflecting everyday activities.

Methods: Forty-three patients with relapsing-remitting MS enrolled in an eye-tracking experiment consisting of a visual search task with naturalistic images. Patients were grouped into "impaired" and "unimpaired" according to their SDMT performance. Reaction time, accuracy and eye-tracking parameters were measured.

Results: The groups did not differ regarding age, gender, and visual acuity. Patients with impaired SDMT (cut-off SDMT-z-score $<-1.5$ ) performance needed more time to find and fixate the target $(q=0.006)$. They spent less time fixating the target $(q=0.042)$. Impaired patients had slower reaction times and were less accurate (both $q=0.0495$ ) even after controlling for patients' upper extremity function. Exploratory analysis revealed that unimpaired patients had higher accuracy than impaired patients particularly when the announced target was in unexpected location $(p=0.037)$. Correlational analysis suggested that SDMT performance is inversely linked to the time to first fixation of the target only if the announced target was in its expected location $(r=-0.498, p=0.003$ vs. $r=-0.212, p=0.229$ ).

Conclusion: Dysfunctional visual search behavior may be one of the mechanisms translating cognitive deficits into difficulties in everyday tasks in MS patients. Our results suggest that cognitively impaired patients search their visual environment less efficiently and this is particularly evident when top-down processes have to be employed.

Keywords: multiple sclerosis, eye tracking (ET), cognitive impairment (CI), cognition, visual search (VS), everyday tasks 


\section{INTRODUCTION}

Cognitive deficits are associated with unemployment, fewer social contacts as well as problems with household activities, and thus have an essential impact on the daily lives of multiple sclerosis (MS) patients (1-5). However, it can be rather challenging to understand how poor performance on a neuropsychological test translates into impaired functioning in everyday life if only conventional laboratory measurements with limited relevance to real-life experiences of MS patients are used. Here, we employed eye-tracking analysis during an ecologically valid visual search task to investigate how cognitively impaired MS patients as defined by the diagnostic standard, the Symbol Digit Modalities Test (SDMT) $(3,6)$, differ from those with a preserved information processing ability.

Eye-tracking has the potential to measure cognition in neurodegenerative diseases (7) and requires only a limited amount of resources. It will become more widespread in the foreseeable future, as the quality of cameras in laptops and tablets and the necessary software has developed to such an extent making it possible to employ them for cognitive experiments, e.g., via a web browser (8). Studies using eyetracking while performing the SDMT have already shown that MS patients differ from healthy individuals, among other measures, with an increased total number of fixations in the test area (9). This suggests that MS patients may have uncertainties during their visual search behavior, possibly reflected by the fact that the target areas are checked multiple times for safety (9). This might be a direct consequence of their cognitive impairment. Similarly, patients with mild cognitive impairment (MCI), often an early stage of the Alzheimer's disease continuum, have been shown to perform significantly worse than healthy controls in a visual search task, which was associated with more pronounced cognitive impairment $(10,11)$.

However, a typical experimental task using simple visual stimuli (e.g., arrows, dots, etc.) does not reflect a situation from the patients' everyday life. A visual search task with pictures showing everyday situations would possess a much higher ecologically validity. Therefore, we explored eye-tracking during a visual search task with pictures showing everyday scenarios. The decision where and when to move the point of fixation is a key aspect of eye-movement control (12). This decision is driven by visuospatial attention and modulates the speed of visual search (13). In general, attentional top-down control is one of the key aspects in visual search (14) and thus eye-tracking can reflect cognitive processes $(7,9-12)$.

We hypothesized that MS patients with low SDMT scores would need more time to fixate the location of the target object, as this reflects processing speed and the integrity of top-down processes such as expectations and prior knowledge that determine where to search for a target object. In addition, we expected the less efficient search behavior of cognitively impaired patients to be associated also with a larger amount of fixations of non-target areas prior to the first fixation of the target and a shorter total fixation duration compared to cognitively preserved patients.

\section{METHODS}

\section{Study Population}

Forty-three patients with relapsing-remitting multiple sclerosis (RRMS) were enrolled in the study. Four of them did not complete the entire experiment. In addition, three patients were excluded due to a gaze sampling rate below $60 \%$ (percentage of correctly measured eye movements by the eye tracker). Data from the remaining 36 RRMS patients were included in the analysis. Table 1 shows the demographic and clinical characteristics of the sample.

The Ethics Committee of the Medical Faculty of the Goethe University Frankfurt am Main approved this study and informed consent was obtained from each patient. Patients were recruited through the neurology department of the University Hospital in Frankfurt am Main. MS was diagnosed according to the 2010 revision of the McDonald criteria (15). All patients had a corrected visual acuity above 0.5 assessed with a vision chart. A trained neurologist assessed the "Expanded Disability Status Scale" (EDSS).

\section{Design and Data Acquisition}

The study comprised two sessions, which were conducted on two different days to reduce the effect of fatigue on performance. Demographics and visual acuity were recorded during the first session. One half of the patients completed first the SDMT and on another day, not more than 6 weeks later, the eye-tracking session. The other half of the patients completed the tasks in reversed sequence.

\section{Eye-Tracking Design}

To investigate the effect of cognitive impairment on visual search in naturalistic scenes, we employed an ecologically valid visual stimulus collection: the BOiS-Database (16) (for examples see Figure 1). The database includes photographs of natural surroundings with scenes from everyday life (e.g., a refrigerator with an open door). In each picture, we defined a prominent object (e.g., a carton of milk, usually expected to be in the refrigerator) as a target. Thirty-five images with a corresponding target object (e.g., milk carton in the fridge door) were chosen. To keep patients alert, we included also 34 images in which the target object was absent and 34 images in which the target was in an unexpected position (e.g., milk carton on the floor). An area-of-interest (AoI) corresponding to the shape (rectangle, circle, ellipse) of the target object was individually defined for each image with a target object. The AoI was defined as an area 2.5 times the size of the target object (Figure 1). Each trial started with the written presentation of the target object's name (e.g., "milk carton") for 3,000 ms, followed by the presentation of the image for $7,000 \mathrm{~ms}$. The patients were instructed to respond via button press whether the target object was present or absent in the image. Responses were made via a response pad (LogiLink ${ }^{\circledR}$ Keypad).

We used a Tobii Pro X2-60 (Tobii, Danderyd, Sweden) eyetracker with a $60 \mathrm{~Hz}$ sampling rate (binocular) and a maximum total system latency of $<35 \mathrm{~ms}$. For each patient a nine-point calibration was performed. The eye tracker with the external 
TABLE 1 | Demographic and clinical characteristics of the patients.

\begin{tabular}{|c|c|c|c|c|c|c|}
\hline & SDMT z & Unimpaired & Impaired & Sum of squares & $F$-value & $p$-value \\
\hline \multirow[t]{2}{*}{ Age (years) ${ }^{a}$} & $>-1$ vs. $<-1$ & $36.29 \pm 11.54$ & $42.50 \pm 11.33$ & 308.347 & 2.343 & 0.135 \\
\hline & $>-1.5$ vs. $<-1.5$ & $36.44 \pm 11.23$ & $44.11 \pm 11.77$ & 396.750 & 3.076 & 0.088 \\
\hline \multirow[t]{2}{*}{ Visual acuity ${ }^{a}$} & $>-1$ vs. $<-1$ & $0.82 \pm 0.15$ & $0.76 \pm 0.16$ & 0.036 & 1.500 & 0.229 \\
\hline & $>-1.5$ vs. $<-1.5$ & $0.83 \pm 0.15$ & $0.73 \pm 0.16$ & 0.062 & 2.686 & 0.110 \\
\hline \multirow[t]{2}{*}{ Disease duration (years) ${ }^{a}$} & $>-1$ vs. $<-1$ & $5.33 \pm 6.12$ & $10.31 \pm 9.20$ & 198.204 & 3.763 & 0.061 \\
\hline & $>-1.5$ vs. $<-1.5$ & $5.49 \pm 6.12$ & $11.49 \pm 9.83$ & 243.240 & 4.737 & 0.037 \\
\hline \multirow[t]{2}{*}{ EDSS $^{a}$} & $>-1$ vs. $<-1$ & $1.81 \pm 1.08$ & $3.33 \pm 1.68$ & 18.503 & 10.833 & 0.002 \\
\hline & $>-1.5$ vs. $<-1.5$ & $1.91 \pm 1.30$ & $3.56 \pm 1.33$ & 18.336 & 10.704 & 0.002 \\
\hline \multirow[t]{2}{*}{ 9HPT (seconds) ${ }^{a}$} & $>-1$ vs. $<-1$ & $19.79 \pm 3.27$ & $29.13 \pm 7.82$ & 696.889 & 25.796 & $<0.000$ \\
\hline & $>-1.5$ vs. $<-1.5$ & $20.65 \pm 4.50$ & $29.67 \pm 8.22$ & 549.002 & 17.504 & $<0.000$ \\
\hline \multirow[t]{2}{*}{ SDMT (z score $)^{\mathrm{a}}$} & $>-1$ vs. $<-1$ & $-0.08 \pm 0.64$ & $-2.03 \pm 0.64$ & 30.135 & 72.791 & $<0.000$ \\
\hline & $>-1.5$ vs. $<-1.5$ & $-0.21 \pm 0.71$ & $-2.28 \pm 0.51$ & 28.871 & 63.995 & $<0.000$ \\
\hline \multirow[t]{2}{*}{ Gender $^{b}$} & $>-1$ vs. $<-1$ & $20 \mathrm{f}, 4 \mathrm{~m}$ & $7 \mathrm{f}, 5 \mathrm{~m}$ & - & - & 0.126 \\
\hline & $>-1.5$ vs. $<-1.5$ & $22 \mathrm{f}, 5 \mathrm{~m}$ & $5 \mathrm{f}, 4 \mathrm{~m}$ & - & - & 0.184 \\
\hline
\end{tabular}

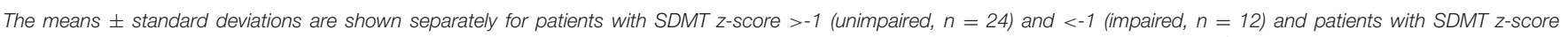

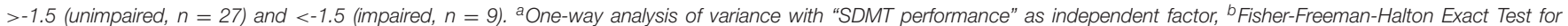
contingency tables.

F, female; m, male; EDSS, expanded disability status scale; SDMT, Symbol Digit Modalities Test. Bold values indicate significant values.

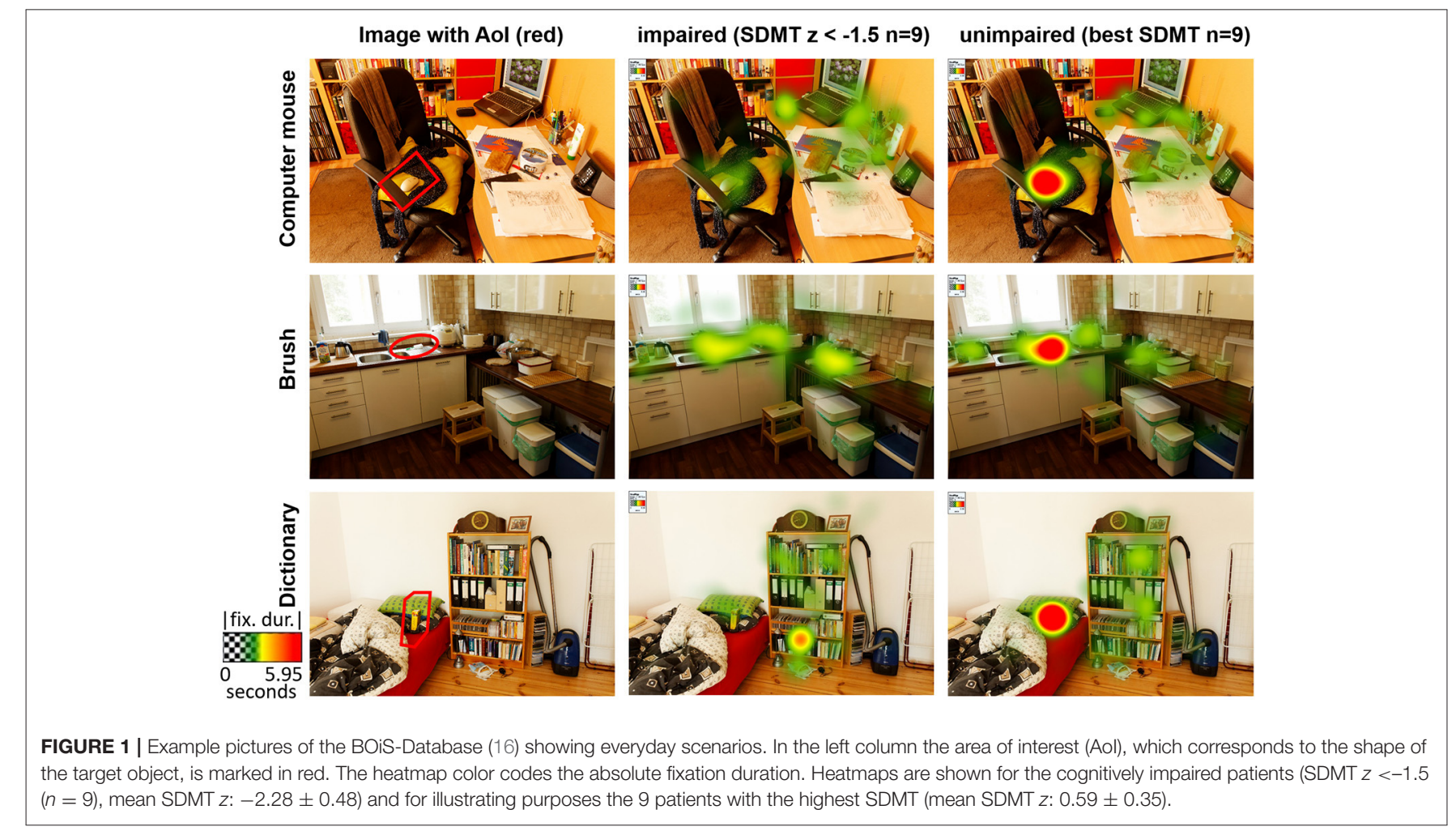

processing unit was connected to the recording notebook (Dell Inspirion 7559-0092, Intel ${ }^{\circledR}$ Core $^{\mathrm{TM}}$ i7-6700HQ) running Tobii Pro Studio (Version 3.4.8.1348, Tobii, Danderyd, Sweden). The eye tracker was placed on the lower end of the display of the recording notebook. The screen had a resolution of $1.920 \times$ 1.080 pixels and a diagonal of $39.6 \mathrm{~cm}$. We used standardized room lighting.
Tobii Studio was used to calculate the eye tracking parameters of interest in trials where the target object was correctly identified as present. Fixations were calculated using the fixation filter implemented in Tobii Studio. This algorithm assumes that the eyes move between fixation points, and therefore detects fixations when a segment of the eye-tracking signal is constant or changing slowly due to drift, or when there is an abrupt change in the 
signal indicating that the eyes have moved to a different fixation position. Using those parameters the "time to first fixation" (TFF), "fixations before" (FB), "total fixation duration" (TFD), and "fixation count" (FC) for the AoI were calculated separately for each picture in which the target object was presented. The TFF describes the latency until the expected target position was fixated for the first time. FB gives the number of fixation points before the AoI was fixated the first time. The TFD describes how long the target object was fixated. FC gives the number of fixations on the AoI.

MATLAB (2013b, Natick, Massachusetts: The MathWorks Inc.) was used to prepare the data for further statistical analyses. The mean and standard deviation per patient of the eye-tracking parameters in trials with correct responses were calculated. Additionally the accuracy as a percentage of correct manual responses (button presses) and the corresponding reaction times were calculated.

\section{Statistical Analysis Sample Size Calculations}

Sample size calculations were computed based on the approach described in Hulley et al. (17) and Chow et al. (18) and the website www.sample-size.net. For this purpose, a proportion of $30 \%$ cognitively impaired MS patients was assumed (19). Furthermore, we employed the visual search parameter means of MS patients and cognitively healthy individuals as well as the standard deviations reported in Utz et al. (20), who utilized a visual search paradigm and neuropsychological tests to discriminate MS patients from healthy controls. Using the T-statistic and non-centrality parameter, a total sample size of $N=35$ ( $n=11$ cognitively impaired and $n=24$ cognitively preserved) subjects was calculated.

\section{Neuropsychological Tests}

Using age- and education-normative data, SDMT raw scores were transformed to $z$-scores (6). First, the threshold for below-average SDMT performance was set to a conservative and widely accepted threshold for cognitive impairment of $z<-1.5(19,21,22)$. Additionally, we performed an exploratory analyses with the aim to determine if eye tracking would detect differences even if the threshold for impairment is less conservative at SDMT $z<-1$. Thus, cognitive impaired patients with a $z<-1.5$ were also in the less conservative group (SDMT $<-1)$.

Baseline demographic and clinical characteristics of the groups ("SDMT $z>-1$ vs. SDMT $z<-1$ " and "SDMT $z>-1.5$ vs. SDMT $z<-1.5$ ") were compared using oneway ANOVAs and the Fisher-Freeman-Halton Exact Test for contingency (Table 1). Significance was set to $p<0.05$.

\section{Eye Tracking}

To evaluate the visual search behavior of patients with MS depending on their cognitive status, we computed one-way ANOVAs with the category "SDMT performance" (" $z<-1.5$ vs. $z \geq-1.5$ " and " $z<-1$ vs. $z \geq-1$," respectively), as independent variable, while "time to first fixation" (TFF), "fixations before" (FB), "total fixation duration" (TFD), "fixation count" (FC), and "accuracy" (ACC) were used as dependent variables in each of the
ANOVAs. To take into account the significantly different upper extremity motor functions of the two groups, we modeled the average 9-hole peg test (9HPT) performance for the dominant hand as a covariate in the analysis of reaction time. Results for our main analyses (SDMT $z<-1.5$ ) were corrected for falsediscovery rate (FDR) using the Benjamin-Hochberg procedure. Adjusted and corrected for multiple comparisons $q$-values were calculated and significance level was set at $q<0.05$. The significance for our exploratory analyses (SDMT $z<-1$ ) was set to $p<0.05$.

Furthermore, we performed an exploratory analysis where unimpaired (SDMT $z$-score $>-1.5$ ) and impaired (SDMT $z$-score $<-1.5)$ MS patients were compared using repeated measurements general linear models with the corresponding eye tracking or performance parameters (TFF, FB, TFD, FC, accuracy, reaction time) as dependent variables, expectedness of the target (expected vs. unexpected position of the target) as within-subjects factor and SDMT performance (unimpaired vs. impaired) as between-subjects factor. Additionally, for reaction time the average 9HPT performance for the dominant hand was included as a covariate. Pearson correlations were computed between SDMT and eye-tracking parameters, accuracy, and reaction times for each of the two conditions expected vs. unexpected. The significance for this exploratory analysis was set to $p<0.05$.

All statistical analyses were performed using SPSS (IBM Corp. Released 2013. IBM SPSS Statistics for Windows, Version 22.0. Armonk, NY: IBM Corp.).

\section{RESULTS}

When using $z<-1$ as a cut-off for impaired performance in SDMT, impaired and unimpaired MS patients did not differ regarding age, gender, visual acuity and disease duration (all $p$ $>0.05$, Table 1). The same was found when using $z<-1.5$ as a threshold (all $p>0.05$, Table 1). However, in this analysis impaired patients exhibited a longer disease duration $(p=0.037$, Table 1). For both definitions of below-average information processing speed, impaired patients had a higher EDSS value ( $p$ $=0.002)$ and 9-hole-peg-test score $(p<0.001$; Table 1).

Comparing the impaired patients (SDMT $z<-1.5$ ) with cognitively unimpaired patients we observed significant differences on TFF $(p=0.001, q=0.006)$, TFD $(p=0.014$, $q=0.042)$, and ACC $(p=0.033, q=0.0495)$. Patients with good SDMT performance were faster in fixating the target for the first time $(1.55 \mathrm{~s} \pm 0.39 \mathrm{~s}$ vs. $2.06 \mathrm{~s} \pm 0.24 \mathrm{~s})$, fixated the target longer $(2.38 \mathrm{~s} \pm 1.19 \mathrm{~s}$ vs. $1.28 \mathrm{~s} \pm 0.75 \mathrm{~s})$ and were more accurate $(72 \pm 11 \%$ vs. $62 \pm 10 \%)$ in detecting the target (Figure 1, Table 2).

In the explorative analyses of the visual search behavior of MS patients, we found significant effects of their SDMT performance (SDMT $z>-1$ vs. SDMT $z<-1$ ) on TFF ( $p=0.006$ ), TFD $(p=0.005)$, and FC $(p=0.033)$. Patients with average or better SDMT performance were faster in fixating the target for the first time $(1.55 \mathrm{~s} \pm 0.40 \mathrm{~s}$ vs. $1.96 \mathrm{~s} \pm 0.33 \mathrm{~s})$ and they had more $(6.08 \pm$ 
TABLE 2 | Visual search behavior depending on SDMT.

\begin{tabular}{|c|c|c|c|c|c|c|}
\hline & Unimpaired & Impaired & Sum of squares & $F$-value & $p$-value & $q$-value \\
\hline Time to first fixation (seconds) ${ }^{a, d}$ & $1.55 \pm 0.39$ & $2.06 \pm 0.24$ & 1.745 & 13.559 & 0.001 & 0.006 \\
\hline Fixations before ${ }^{a, d}$ & $4.44 \pm 1.04$ & $4.71 \pm 1.44$ & 0.494 & 0.373 & 0.546 & 0.546 \\
\hline Total fixation duration (seconds)a,d & $2.38 \pm 1.19$ & $1.28 \pm 0.75$ & 8.021 & 6.712 & 0.014 & 0.042 \\
\hline Fixation count ${ }^{\mathrm{a}}$ & $5.92 \pm 1.53$ & $4.86 \pm 2.26$ & 7.534 & 2.487 & 0.125 & 0.15 \\
\hline Accuracy $(1 \triangleq 100 \%)^{a}$ & $0.72 \pm 0.11$ & $0.62 \pm 0.10$ & 0.056 & 4.957 & 0.033 & 0.0495 \\
\hline Reaction time (seconds) ${ }^{b}$ & $3.43 \pm 0.58$ & $3.93 \pm 0.61$ & $1.855^{\mathrm{c}}$ & 5.348 & 0.027 & 0.0495 \\
\hline
\end{tabular}

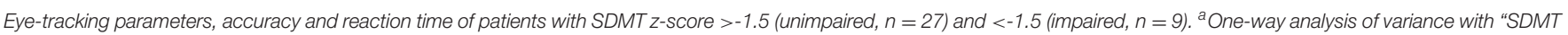

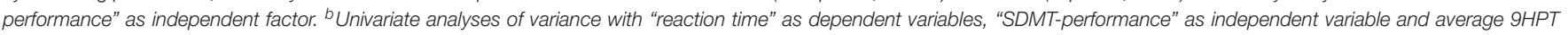

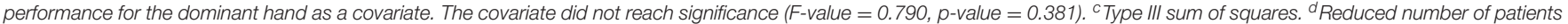
due to missing values ( $n=25$ vs. 9). Adjusted and corrected for multiple comparisons (FDR) $q$-values with significance level at $q<0.05$. Bold values indicate significant values.

TABLE 3 | Visual search behavior depending on SDMT.

\begin{tabular}{|c|c|c|c|c|c|}
\hline & Unimpaired & Impaired & Sum of squares & $F$-value & $p$-value \\
\hline Time to first fixation (seconds) ${ }^{a, d}$ & $1.55 \pm 0.40$ & $1.96 \pm 0.33$ & 1.236 & 8.546 & 0.006 \\
\hline Fixations before ${ }^{a, d}$ & $4.48 \pm 1.04$ & $4.59 \pm 1.37$ & 0.089 & 0.067 & 0.798 \\
\hline Total fixation duration (seconds) ${ }^{a, d}$ & $2.47 \pm 1.19$ & $1.29 \pm 0.71$ & 10.280 & 9.141 & 0.005 \\
\hline Fixation counta,d & $6.08 \pm 1.48$ & $4.71 \pm 2.05$ & 14.082 & 4.985 & 0.033 \\
\hline Accuracy $(1 \triangleq 100 \%)^{a}$ & $0.71 \pm 0.11$ & $0.65 \pm 0.11$ & 0.033 & 2.690 & 0.110 \\
\hline Reaction time (seconds) ${ }^{\mathrm{b}}$ & $3.44 \pm 0.57$ & $3.78 \pm 0.68$ & $1.004^{\mathrm{c}}$ & 2.693 & 0.110 \\
\hline
\end{tabular}

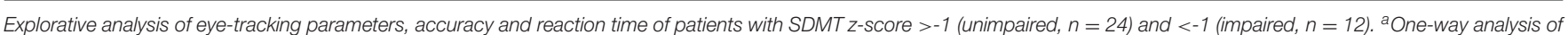

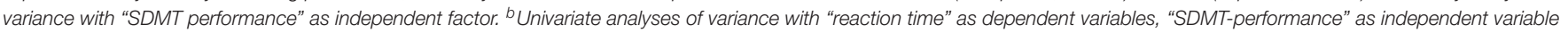

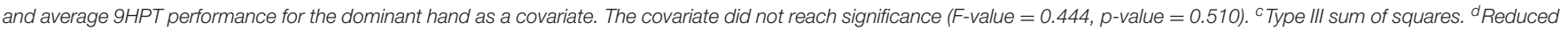
number of patients due to missing values ( $n=23 \mathrm{vs.}$ 11). Bold values indicate significant values.

1.48 vs. $4.71 \pm 2.05)$ and longer $(2.47 \mathrm{~s} \pm 1.19 \mathrm{~s}$ vs. $1.29 \mathrm{~s} \pm 0.71 \mathrm{~s})$ fixations on the target (Table 3 ).

Univariate analyses of variance with "reaction time" as dependent variable, "SDMT performance" as independent variable and the 9HPT as a covariate showed that the main effect of SDMT performance was significant with slower reaction times for the impaired patients (SDMT $z<-1.5)(p=0.027, q=$ $0.0495,3.437 \mathrm{~s} \pm 0.566 \mathrm{~s}$ vs. $3.782 \mathrm{~s} \pm 0.681 \mathrm{~s})$. The $9 \mathrm{HPT}$ as a covariate was not significant $(p=0.381)$. Using the explorative cut-off of SDMT $z<-1$ did not show any significant effect on reaction time and the 9HPT performance $(p=0.110$ and $p$ $=0.510)$.

Including the within-subjects factor "expectedness" as another exploratory analysis revealed an interaction between the expectedness of the location of the target and the cognitive status only for accuracy with unimpaired patients being more accurate than impaired patients for the expected condition, while this difference was even more pronounced for the unexpected condition ( $p=0.037$, Table 4, Figure 2). Similar to our primary analysis, we found for TFF, TFD and reaction time but not for FB and FC significant group differences (see Table 4). Not surprisingly, the main effect of expectedness was significant for almost all eye tracking performance and behavioral measures with stimuli in expected locations resulting in better accuracy, shorter reaction times, less TFF and FB as well as more FC (Table 4). We found also significant correlations between SDMT performance and TFF expected $(r=-0.498, p=0.003$,
Figure 3) but not TFF unexpected $(r=-0.212, p=0.229)$, as well as between SDMT and TFD expected $(r=0.441$, $p=0.009)$ and TFD unexpected $(r=0.484, p=0.004)$, (see Figure 3).

\section{DISCUSSION}

Our findings demonstrate how impaired cognitive processing speed in MS is translated directly into dysfunctional visual search in naturalistic scenes measured with eyetracking. Impaired patients take longer to find and fixate the target object, and although they take longer, they are less accurate.

They fixated the target object for a shorter time, probably because they first needed more time to locate it in the scene and invested thus more time in exploring the periphery to gain confidence about their decision. The SDMT is diagnostically helpful, but it does not tell us anything about the real world. Our results offer an explanation how impaired cognitive processing limits the patients' daily functioning.

Impaired patients (SDMT $z<-1.5$ ) indicated with less accuracy whether the searched object was present or not and needed more time to react. This difference was even more pronounced, as demonstrated by the significant GLM interaction for accuracy, when the target was located in an unexpected position (Figure 2). This suggests that visual search in naturalistic scenes is strongly driven by top-down cognitive processes. Our 
TABLE 4 | Visual search behavior depending on SDMT and expectedness of the location of the target.

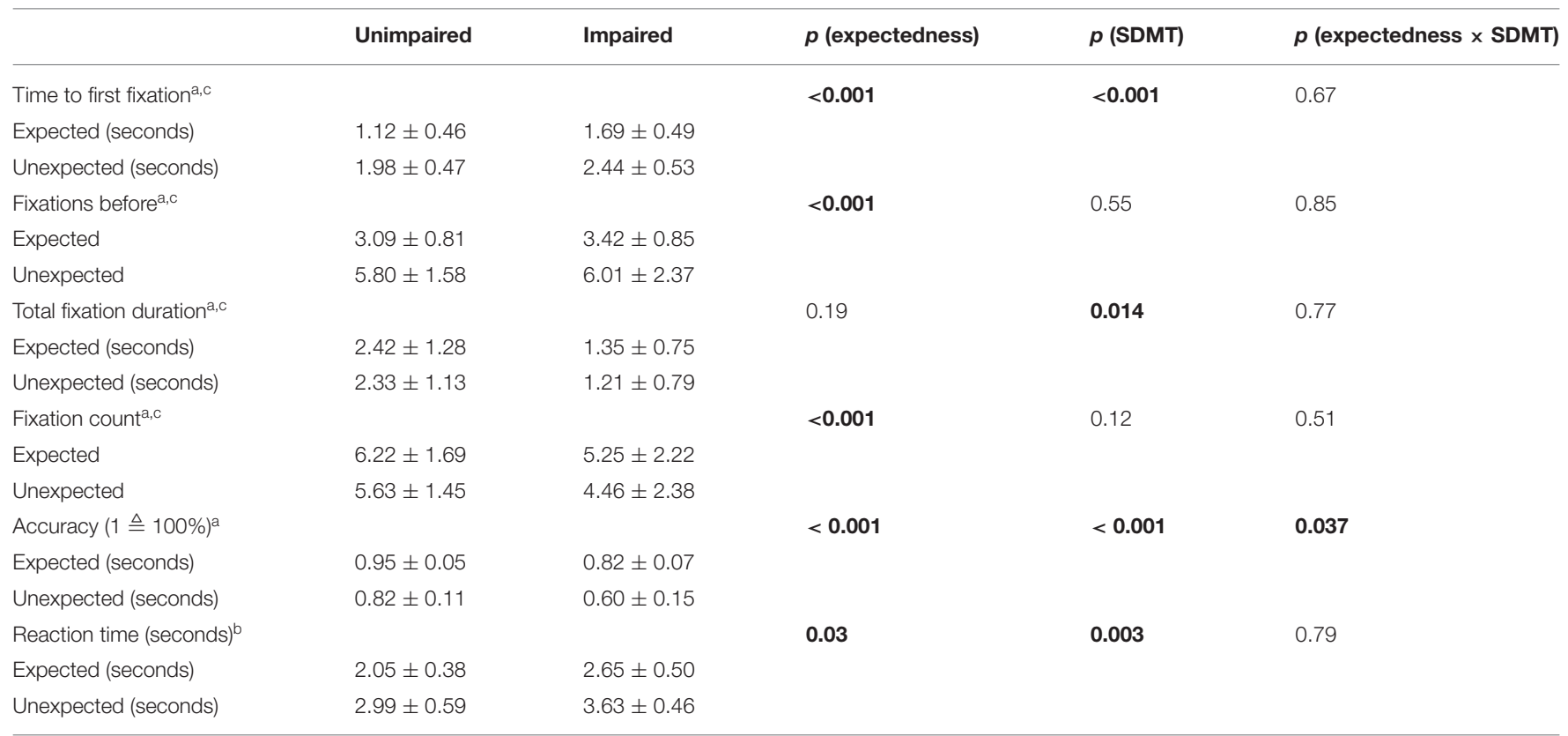

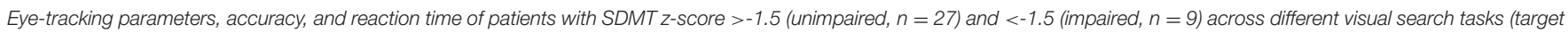

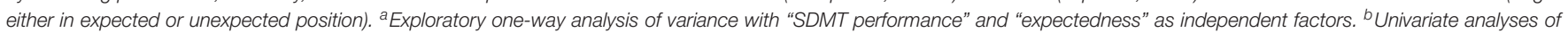

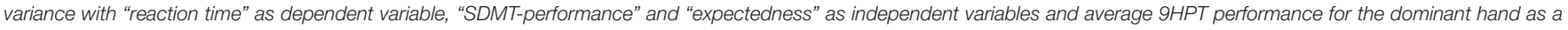

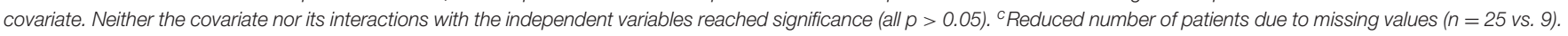
Bold values indicate significant values.

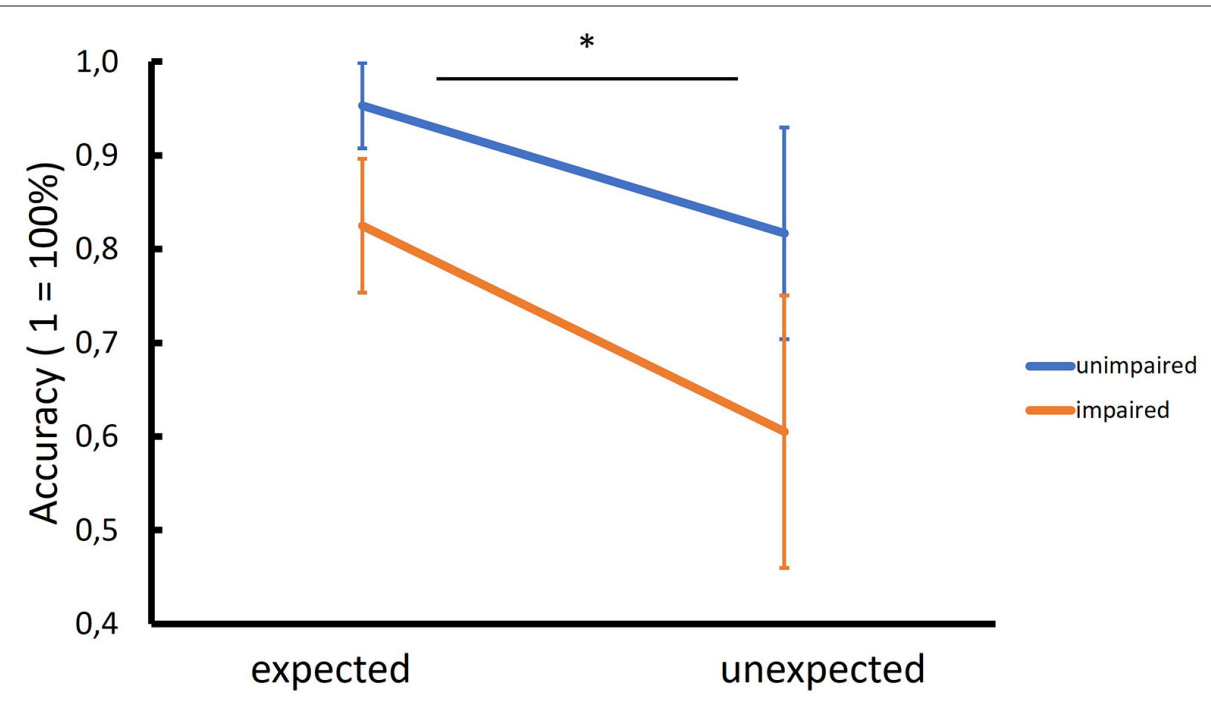

FIGURE 2 | Interaction between cognitive status and expectedness of the location of the target. While cognitively unimpaired MS patients had higher accuracy than impaired patients to detect targets in their expected positions (e.g., milk carton in the fridge), this difference was even more pronounced when the target was in an unexpected position (e.g., milk carton on the floor) $\left(^{*}=\right.$ significant GLM interaction, $p=0.037$ ).

patients were instructed before each trial which object they had to search for. An intact information processing ability would allow the subject to optimize his or her searching strategy (e.g., "milk carton is usually found in the fridge") and fixate the target more quickly, if it is located in its expected position, as demonstrated by the significant correlation between SDMT performance and time to first fixation for the expected condition (Figure 3). This, however, seems not to be the case, if the target is in an unexpected position (e.g., on the floor)-in this case optimizing the search strategy is not possible by employing top-down cognitive processes, as evident by the absence of correlation between SDMT 


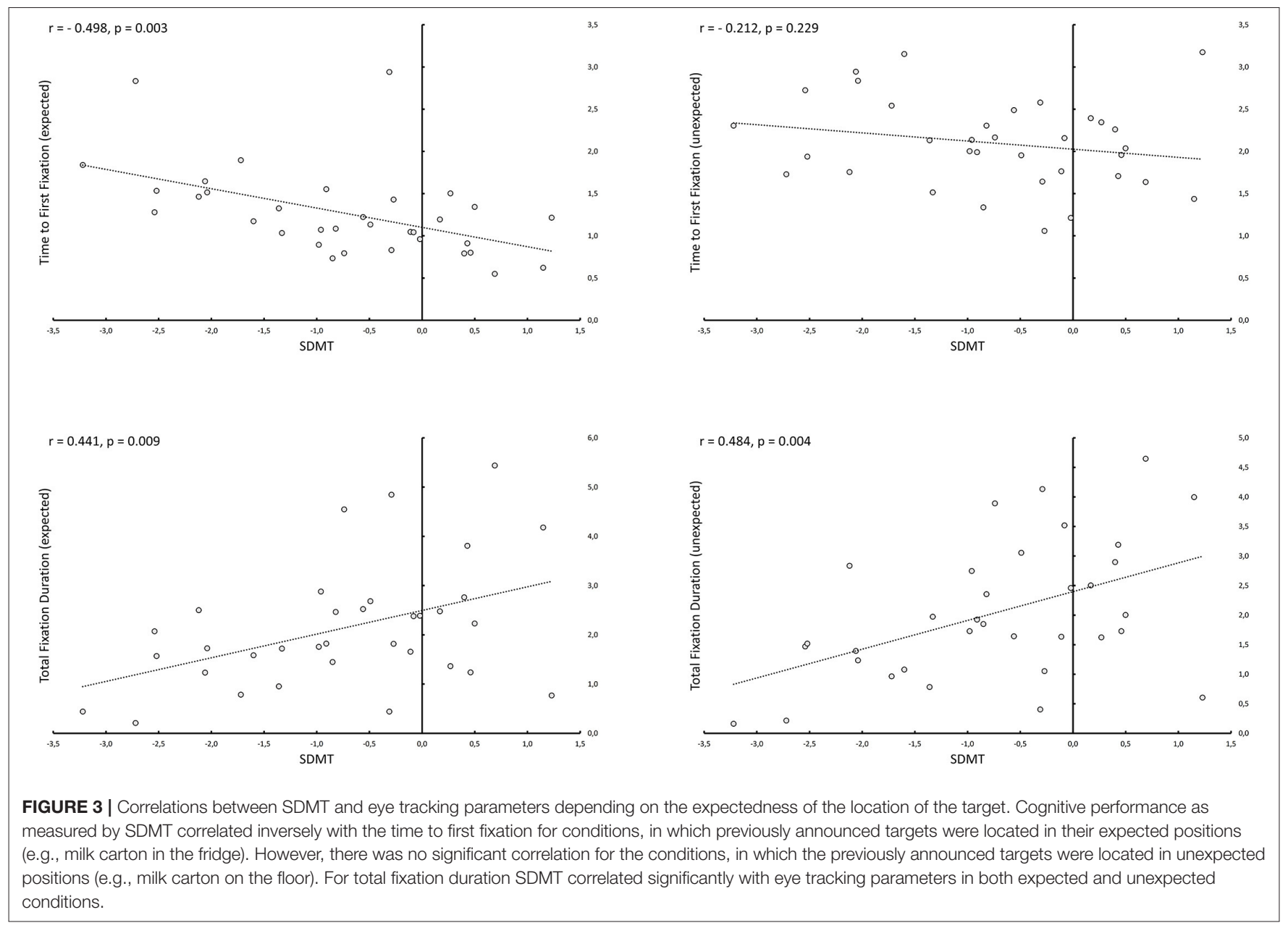

performance and time to first fixation for the unexpected condition (Figure 3).

Interestingly, when using a more permissive threshold for defining cognitive impairment (SDMT $z<-1$ ), group differences in visual search behavior remained significant while behavioral measures (i.e., accuracy and reaction time) were no longer significant. This suggests that eye-tracking could be useful in detecting subtle cognitive impairment even before neuropsychological and behavioral performance declines.

Using saccade analysis, previous work has shown that MS patients have a higher error rate (e.g., looking to the wrong side) and a longer latency compared to healthy controls (23). In particular, SDMT and the "Paced Auditory Serial Addition Test" performance correlate with error rate and latency (24). Our study relates to these findings, even though we did not explicitly study saccades. Saccades and fixations are the basis of eye movements (12) and TFF reflects the combined time from all saccades and fixations before the first fixation on the target and thus provides at least an indirectly comparable measure. Thus, our results complement the current literature by employing an ecologically valid paradigm (naturalistic visual scenes) and experimental instructions that are closer to the real challenges MS patients face in their daily lives. Since saccade generation is essential for an optimal performance in a visual search task, the increased error rate found in previous studies using saccade measurements might be linked to our results showing lower accuracy of MS patients. Furthermore, longer latencies in saccade generation might be associated with longer search times (25), which are increased in MS, as we show here using TFF.

It is not surprising that there is a correlation between the SDMT and eye-tracking, as the SDMT assesses visual scanning, memory and perceptual speed, among other functions (26). Pavisian et al. demonstrated that the eye-tracking behavior of MS patients differs from that of healthy individuals during the performance of the SDMT (9). We extend these findings to a more naturalistic task that is directly linked to the patients' daily lives and stress the importance of intact information processing speed for visual search in the context of real-life situations.

With increasing availability of eye-tracking, e.g., based on web browsers, visual screening tests could be performed, for example before an appointment at the doctor's office or even at home. They could be used to identify patients who should undergo a more intensive neuropsychological examination. Furthermore, understanding better how daily functions are affected by impaired information processing could facilitate the development of better cognitive monitoring and rehabilitation 
strategies. Our results could be explained on the one hand by slowed bottom-up information processing and on the other hand by impaired top-down executive control. Since both perceptual processes and executive control are impaired in MS (27-29), it remains unclear to what extent deficits of bottom-up or topdown processing or their combination are causal for the cognitive deficits of MS patients.

In MS, but also in other neurodegenerative diseases, eye tracking has limitations in cases of severely reduced visual acuity or oculomotor dysfunction (involvement of cranial nerves). The significance of this problem is less essential for patients at the early stages of the disease but might become more serious and hamper implementing eye-tracking in cognitive monitoring or rehabilitation of patients with higher EDSS scores or in advanced progressive stages of the disease.

One further possible limitation is the variable delay between the eye tracking experiment and SDMT testing (up to 6 weeks). In order to prevent fatigue, the two measurements were not performed on the same day, although we knew that this could affect our results. However, since the sequence (SDMT first, eye tracking second and vice versa) of the two measurements was pseudorandomized across all participants, we believe that the influence of any possible systematic bias on our findings has been minimized. Nevertheless, future studies should employ, if possible, a standardized measurement schedule.

Among the limitations of the current study are the small to moderate number of patients included, the absence of progressive MS patients and the cross-sectional nature of our investigation. Longitudinal studies with different disease phenotypes would extend our findings and test the notion that eye-tracking changes predict later clinical and/or cognitive decline. Interestingly, a longitudinal study over 2 years studying antisaccades, has shown that error rate, latency and accuracy worsened in MS patients, whereas EDSS scores remained largely unchanged, which might suggest that eye-tracking could be sensitive to subclinical disease activity and more subtle neurodegenerative processes (30).

Naturalistic cognitive assessments which reflect everyday difficulties that MS patients encounter in their daily routine might be challenging. Visual search tools employing ecologically valid paradigms would be one way to address this problem,

\section{REFERENCES}

1. Rao SM, Leo GJ, Ellington L, Nauertz T, Bernardin L, Unverzagt F. Cognitive dysfunction in multiple sclerosis. Neurology. (1991) 41:6926. doi: 10.1212/WNL.41.5.692

2. Chiaravalloti ND, Deluca J. Cognitive impairment in multiple sclerosis. Lancet Neurol. (2008) 7:1139-51. doi: 10.1016/S1474-4422(08) 70259-X

3. Benedict RH, Deluca J, Phillips G, LaRocca N, Hudson LD, Rudick R. Validity of the symbol digit modalities test as a cognition performance outcome measure for multiple sclerosis. Mult Scler. (2017) 23:72133. doi: 10.1177/1352458517690821

4. Brochet B, Ruet A. Cognitive impairment in multiple sclerosis with regards to disease duration and clinical phenotypes. Front Neurol. (2019) 10:261. doi: 10.3389/fneur.2019.00261 but data supporting their validation in MS is limited. On the other hand, there is an increasing amount of data using e.g., cell phone applications or wearables $(31,32)$ to detect disease progression parameters. After appropriate validation, eye-tracking employed by such applications might prove useful in identifying progression in cognitive impairment.

\section{DATA AVAILABILITY STATEMENT}

The raw data supporting the conclusions of this article will be made available by the authors, without undue reservation.

\section{ETHICS STATEMENT}

The studies involving human participants were reviewed and approved by Ethikkommission des Fachbereichs Medizin, Faculty of Medicine, Goethe-University Frankfurt. The patients/participants provided their written informed consent to participate in this study.

\section{AUTHOR CONTRIBUTIONS}

JG: designed and conceptualized study, analyzed and interpreted the data, and drafted and revised the manuscript for intellectual content. HB and LF: analyzed the data, interpreted the data, and major role in the acquisition of data. DS and $\mathrm{CB}$ : analyzed the data and major role in the acquisition of data. MN, JK, SF, and MB: designed and conceptualized study, interpreted the data, and revised the manuscript for intellectual content. CF: designed and conceptualized study and revised the manuscript for intellectual content. YY: design and conceptualized study, analyzed and interpreted the data, and drafted and revised the manuscript for intellectual content. All authors contributed to the article and approved the submitted version.

\section{ACKNOWLEDGMENTS}

We thank the team of the Multiple Sclerosis Outpatient Clinic of the University Hospital Frankfurt.
5. Srpova B, Sobisek L, Novotna K, Uher T, Friedova L, Vaneckova M, et al. The clinical and paraclinical correlates of employment status in multiple sclerosis. Neurol Sci. (2021) 1-10. doi: 10.1007/s10072-021-05 553-z. [Epub ahead of print].

6. Smith A. Symbol Digit Modalities Test. Los Angeles, CA: Western Psychological Services (1982).

7. Bueno APA, Sato JR, Hornberger M. Eye tracking - the overlooked method to measure cognition in neurodegeneration? Neuropsychologia. (2019) 133:107191. doi: 10.1016/j.neuropsychologia.2019.107191

8. Semmelmann K, Weigelt S. Online webcam-based eye tracking in cognitive science: a first look. Behav Res. (2018) 50:45165. doi: 10.3758/s13428-017-0913-7

9. Pavisian B, Patel VP, Feinstein A. Cognitive mediated eye movements during the SDMT reveal the challenges with processing speed faced by people with MS. BMC Neurol. (2019) 19:340. doi: 10.1186/s12883-019-1543-8 
10. Tales A, Bayer AJ, Haworth J, Snowden RJ, Philips M, Wilcock G. Visual search in mild cognitive impairment: a longitudinal study. J Alzheimers Dis. (2011) 24:151-60. doi: 10.3233/JAD-2010-101818

11. Pereira MLGDF, Camargo MVZDA, Bellan AFR, Tahira AC, Dos Santos B, Dos Santos J, et al. Visual search efficiency in mild cognitive impairment and alzheimer's disease: an eye movement study. J Alzheimers Dis. (2020) 75:261-75. doi: 10.3233/JAD-190690

12. Liversedge SP, Findlay JM. Saccadic eye movements and cognition. Trends Cogn Sci. (2000) 4:6-14. doi: 10.1016/S1364-6613(99)01418-7

13. Greenwood PM, Parasuraman R. Scale of attentional focus in visual search. Percept Psychophys. (1999) 61:837-59. doi: 10.3758/BF03206901

14. Eimer M. The neural basis of attentional control in visual search. Trends Cogn Sci. (2014) 18:526-35. doi: 10.1016/j.tics.2014.05.005

15. Polman CH, Reingold SC, Banwell B, Clanet M, Cohen JA, Filippi M, et al. Diagnostic criteria for multiple sclerosis: 2010 revisions to the McDonald criteria. Ann Neurol. (2011) 69:292-302. doi: 10.1002/ana.22366

16. Mohr J, Seyfarth J, Lueschow A, Weber JE, Wichmann FA, Obermayer K. BOiS-Berlin object in scene database: controlled photographic images for visual search experiments with quantified contextual priors. Front Psychol. (2016) 7:749. doi: 10.3389/fpsyg.2016.00749

17. Hulley S, Cummings S, Browner W, Grady D, Newman T. Designing Clinical Research: An Epidemiologic Approach. 4th edn. Philadelphia: Lippincott Williams \& Wilkins (2013).

18. Chow SC, Shao J, Wang H. Sample Size Calculations in Clinical Research. 2nd edn. Boca Raton, FL: Chapman \& Hall/CRC (2008)

19. Sumowski JF, Benedict R, Enzinger C, Filippi M, Geurts JJ, Hamalainen P, et al. Cognition in multiple sclerosis: state of the field and priorities for the future. Neurology. (2018) 90:278-88. doi: 10.1212/WNL.0000000000004977

20. Utz KS, Hankeln TMA, Jung L, Lämmer A, Waschbisch A, Lee H, et al. Visual search as a tool for a quick and reliable assessment of cognitive functions in patients with multiple sclerosis. PLOS ONE. (2013) 8:e81531. doi: 10.1371/journal.pone.0081531

21. Filser M, Schreiber H, Pöttgen J, Ullrich S, Lang M, Penner IK. The brief international cognitive assessment in multiple sclerosis (BICAMS): results from the German validation study. J Neurol. (2018) 265:258793. doi: 10.1007/s00415-018-9034-1

22. Hechenberger S, Helmlinger B, Ropele S, Pirpamer L, Bachmaier G, Damulina A, et al. Information processing speed as a prognostic marker of physical impairment and progression in patients with multiple sclerosis. Mult Scler Relat Disord. (2021) 44:103353. doi: 10.1016/j.msard.2021.103353

23. Fielding J, Kilpatrick T, Millist L, White O. Antisaccade performance in patients with multiple sclerosis. Cortex. (2009) 45:900-3. doi: 10.1016/j.cortex.2009.02.016

24. Fielding J, Kilpatrick T, Millist L, White O. Multiple sclerosis: cognition and saccadic eye movements. J Neurol Sci. (2009) 277:32-6. doi: 10.1016/j.jns.2008.10.001

25. Ipata AE, Gee AL, Goldberg ME, Bisley JW. Activity in the lateral intraparietal area predicts the goal and latency of saccades in a free-viewing visual search task. J Neurosci. (2006) 26:3656-61. doi: 10.1523/JNEUROSCI.5074-05. 2006
26. Sheridan LK, Fitzgerald HE, Adams KM, Nigg JT, Martel MM, Puttler LI, et al. Normative symbol digit modalities test performance in a community-based sample. Arch Clin Neuropsychol. (2006) 21:23-8. doi: 10.1016/j.acn.2005.07.003

27. Nebel K, Wiese H, Seyfarth J, Gizewski ER, Stude P, Diener C, et al. Activity of attention related structures in multiple sclerosis patients. Brain Res. (2007) 1151:150-60. doi: 10.1016/j.brainres.2007.03.007

28. Vázquez-Marrufo M, Galvao-Carmona A, González-Rosa JJ, Hidalgo-Muñoz AR, Borges M, Ruiz-Peña JL, et al. Neural correlates of alerting and orienting impairment in multiple sclerosis patients. PLoS ONE. (2014) 9:e97226. doi: 10.1371/journal.pone.0097226

29. Veréb D, Tóth E, Bozsik B, Király A, Szab,ó N, Kincses B, et al. Altered brain network function during attention-modulated visual processing in multiple sclerosis. Mult Scler. (2021) 27:1341-9. doi: 10.1177/13524585209 58360

30. Fielding J, Kilpatrick T, Millist L, Clough M, White O. Longitudinal assessment of antisaccades in patients with multiple sclerosis. PLoS ONE. (2012) 7:e30475. doi: 10.1371/journal.pone.0030475

31. Matthews PM, Block VJ, Leocani L. E-health and multiple sclerosis. Curr Opin Neurol. (2020) 33:271-6. doi: 10.1097/WCO.0000000000000823

32. Alexander S, Peryer G, Gray E, Barkhof F, Chataway J. Wearable technologies to measure clinical outcomes in multiple sclerosis: a scoping review. Mult Scler. (2021) 27:1643-56. doi: 10.1177/1352458520946005

Conflict of Interest: YY has been supported by travel grants from Novartis and Sanofi Genzyme, has received an honorarium for active participation in an advisory board by Sanofi Genzyme as well as speaking honoraria by Roche and Sanofi Genzyme. CF reports speaker honoraria and honoraria for participating in advisory boards from Alexion, Bristol Myers Sqibb, Novartis, Teva, Merck, Sanofi-Genzyme, and Roche. CF received research support from Novartis and Sanofi-Genzyme.

The remaining authors declare that the research was conducted in the absence of any commercial or financial relationships that could be construed as a potential conflict of interest.

Publisher's Note: All claims expressed in this article are solely those of the authors and do not necessarily represent those of their affiliated organizations, or those of the publisher, the editors and the reviewers. Any product that may be evaluated in this article, or claim that may be made by its manufacturer, is not guaranteed or endorsed by the publisher.

Copyright (C) 2022 Gehrig, Bergmann, Fadai, Soydaş, Buschenlange, Naumer, Kaiser, Frisch, Behrens, Foerch and Yalachkov. This is an open-access article distributed under the terms of the Creative Commons Attribution License (CC BY). The use, distribution or reproduction in other forums is permitted, provided the original author(s) and the copyright owner(s) are credited and that the original publication in this journal is cited, in accordance with accepted academic practice. No use, distribution or reproduction is permitted which does not comply with these terms. 